\title{
"The skeletal muscle in chronic respiratory diseases", summary of the ERS Research Seminar in Rome, Italy, February 11-12 1999
}

\author{
P. Palange*, P.D. Wagner**
}

The first Research Seminar sponsored by the European Respiratory Society (ERS), and organized by the "Pulmonary circulation, gas exchange and exercise" scientific group, brought together small groups of investigators to discuss current knowledge in the research field of "Skeletal muscle dysfunction in chronic respiratory diseases". This is currently an issue of much interest on both sides of the Atlantic [1], and has been fueled by repeated observations that in chronic respiratory disorders exercise limitation cannot be explained by ventilatory limitation alone and, more recently, transplantation of diseased lungs does not result in restoration of exercise capacity. Even prior to any kind of surgery, there are data to suggest that in patients with chronic obstructive lung disease (COPD) the skeletal muscles contribute to exercise limitation, and that the lungs, despite their inability to permit high levels of ventilation during exercise, are not the whole story. Thus, evidence of reduced muscle oxidative enzyme levels, of early lactate appearance and of ${ }^{31}$ phosphorous (P)-magnetic resonance spectroscopy (MRS) based high energy phosphate/intracellular $\mathrm{pH}$ differences from normal all suggest muscle abnormalities per se. Impaired muscle oxygen $\left(\mathrm{O}_{2}\right)$ transport may also occur, and separate from the pulmonary abnormalities, add to exercise limitation. Several confounding factors make elucidation of the role of the muscles in exercise limitation difficult to unravel. This is particularly so because of the highly integrated nature of exercise involving basically all organ systems and also because of the multidisciplinary nature of the events that underlie exercise, encompassing physical, biochemical, physiological, and structural principles. These confounding factors include: 1) complex cardiopulmonary regulation of blood flow, ventilation and exercise that may prevent cardiac output from rising above normal to compensate for inadequate $\mathrm{O}_{2}$ transport; 2) difficulty interpreting $\mathrm{O}_{2}$-dependent metabolic parameters in muscle when $\mathrm{O}_{2}$ transport is impaired; 3 ) difficulty knowing how much metabolic impairment is required to reduce exercise capacity; 4) variability in patient characteristics within and between studies; 5) drug treatments with agents that can affect muscles; 6) intercurrent disease; 7) problems in selecting the correct control subjects by which to judge patient responses; 8) effects of muscle mass reduction; 9) the issue of how much of the so-called myopathy is simply due to inactivity.

The overall purpose of the seminar was to explore where the collective understanding of skeletal muscle function in respiratory disease currently stands, and consequently, to lay out the problems to be solved in the future. Interest in the area was indicated by the fact that 59 scientists from 14 countries, experts in the fields of $\mathrm{O}_{2}$ transport, muscle physiology, nutrition, metabolic biochemistry, clinical and molecular approaches to research, and rehabilitation attended the seminar. Only eight of these were actual meeting faculty. The meeting was organized with short introductory lectures and therefore a very large space for the discussion. Twenty-two young researchers were selected to lead and keep alive the discussion of selected topics. The 2-day programme was divided into four distinct sessions, each one addressing specific issues: section 1) exercise limitation in chronic respiratory diseases; section 2) structural and biochemical alterations of skeletal muscles in chronic respiratory diseases; section 3 ) effect of hypoxia and poor nutritional state on skeletal muscle function; section 4) therapeutic interventions on skeletal muscle function in chronic respiratory disorders. Each session lasted $4 \mathrm{~h}$ with $>2 \mathrm{~h}$ devoted to the discussion. The mix of short formal presentation followed by open discussion with discussion leaders allowed for a large participation and great interaction. The format was very appreciated by participants who, at the end of meeting, agreed to keep discussing the area by means of: 1) organizing workshops every other year at the annual ERS meeting and 2) developing a chat page on the ERS Website. The new scientific group, "Foro

\footnotetext{
*Dipartimento di Medicina Clinica, University of Rome "La Sapienza", Italy. **Dept of Medicine, University California San Diego, La Jolla, CA, USA

Faculty: P. Palange (co-chair; I), P.D. Wagner (co-chair; USA), A.G.N. Agusti (E), R. Casaburi (USA), X. Leverve (F), F. Maltais (CDN), J. Roca (E), A.M.W.J. Schols (NL).

Participants: Z Aisanov (RUS), E. Baarends (NL), C. Barbara (PT), A. Bellone (I), M. Bonsignore (I), X. Busquets (E), S. Carlone (I), F. Costes (F), J. Dolensky (CZ), M. Engelen (NL), G. Fiorenzano (I), S. Forte (I), G. Gayan-Ramirez (B), J. Gea (E), B. Grassi (I), M. Gorini (I), H. Gosker (NL), T.L. Griffiths (UK), M. Gudjonsdottir(I), M.C. Hogan (USA), S. Ignjatovic (YUG), E. Kalmanova (RUS), F. Laghi (USA), E. Lampert (F), L. Lands (CDN), P. LeBlanc (CDN), D. MacLeod (UK), E. Marchand (B), R. Marrades (E), C. Mollica (I), J.A. Neder (BRA), V. Novel-Chate (F), P. Onorati (I), A. Patessio (I), K. Peers (B), C. Pison (F), L. Puente-Maestu (E), S. Revill (UK), R.S. Richardson (USA), E. Sala (E), C. Santoriello (I), A. Satta (I), J. Sauleda (E), G. Schmid (I), A. Sebastiani (I), P. Serra (I), A. Somfay (H), T. Storer (USA), W. Stringer (USA), T. Trooster (B), B. Vagaggini (I).
} 
Romano", will have the opportunity to share current concepts and ideas on the role of skeletal muscle dysfunction in chronic lung diseases.

A brief summary of the 2-day meeting with the main conclusions is now reported.

\section{February 11, 1999. Section 1: exercise limitation in chronic respiratory diseases}

The session was opened with a lecture by A.G.N. Agusti (Mallorca, Spain) focusing on the mechanisms that limit exercise tolerance in patients with chronic respiratory diseases and on the tools, old and new, used to investigate them. A.G.N. Agusti reviewed the central and the peripheral causes of exercise limitation in COPD and available tools to investigate the relative contribution of each factors. Among them, the flow-volume curve for the assessment of airflow limitation [2], the multiple inert gas technique for the evaluation of pulmonary gas exchange [3], the measurement of femoral blood flow by thermodilution [4], the kinetics of oxygen uptake during exercise for the evaluation of muscle aerobic capacity [5], the intracelullar metabolic events by nuclear magnetic resonance [6] or near-infrared spectroscopy [7] and the role of skeletal muscle biopsy [8]. A.G.N. Agusti pointed out that the proper evaluation of reduced exercise tolerance in patients with chronic respiratory failure requires an integrative approach that takes into account all potential limiting factors into a multidimensional picture [9].

The effects of hypoxaemia on $\mathrm{O}_{2}$ transport mechanisms in normal and pathological states were presented by P.D. Wagner (LaJolla, CA, USA). Arterial hypoxaemia, caused by chronic respiratory diseases, is associated with a reduction in the rate of delivery of $\mathrm{O}_{2}$ to muscles. In some patients, hypoxaemia may worsen with exercise, compounding the problem of $\mathrm{O}_{2}$ delivery. Additional deleterious effects of arterial hypoxaemia may be seen, during exercise, at the level of the skeletal muscles. Specifically, if exercise is limited by $\mathrm{O}_{2}$ availability rather than by metabolic capacity to use $\mathrm{O}_{2}$ reducing the oxygen tension in arterial blood $\left(\mathrm{Pa}, \mathrm{O}_{2}\right)$ will cause muscle capillary $\mathrm{Pa}, \mathrm{O}_{2}$ to be reduced; this in turn will lower the driving $P \mathrm{a}_{2} \mathrm{O}_{2}$ gradient between the capillaries and the mitochondria, and as a result, the diffusional transport of $\mathrm{O}_{2}$ within muscle will be impaired. Thus, $\mathrm{O}_{2}$ availability to mitochondria is affected by arterial hypoxaemia not only directly (reduced convective $\mathrm{O}_{2}$ delivery) but also indirectly (reduced diffusional transport in muscle). On top of these consequences of COPD, there may be structural changes in muscle as a result of the disease process itself, perhaps again from chronic hypoxaemia. Prolonged muscle inactivity may also contribute to these structural changes. Such changes may be complex, but for $\mathrm{O}_{2}$ transport perhaps the most important relate to reduced muscle capillarity. If the number of capillaries is reduced, a given rate of blood flow may lead to a lower average red cell transit time and thus reduced $\mathrm{O}_{2}$ unloading. However, since there is evidence that the diffusional conductance for $\mathrm{O}_{2}$ between the muscle red cell and the mitochondria is determined mostly by the capillary surface area, any reduction in capillary number will reduce $\mathrm{O}_{2}$ conductance as well as transit time. As impaired $\mathrm{O}_{2}$ transport and the lung disease itself together limit exercise levels, cardiac output and thus muscle blood flow remain low as well. As a result, there may be incomplete muscle capillary recruitment, further lowering capillary density in a functional sense. This may produce even more impediment to $\mathrm{O}_{2}$ transport because of perfusion/metabolism heterogeneity and reduced perfused capillary surface area for $\mathrm{O}_{2}$ diffusion. P.D. Wagner raised the following points about the role of $\mathrm{O}_{2}$ transport in chronic respiratory diseases to be addressed in the discussion: 1 ) is maximal exercise limited by mitochondrial $\mathrm{O}_{2}$ availability or by mitochondrial metabolic capacity to use $\mathrm{O}_{2}$ ? 2) how much is convective $\mathrm{O}_{2}$ delivery to muscle impaired? 3) how much is diffusional $\mathrm{O}_{2}$ delivery (red blood cell (RBC) to mitochondria) impaired? 4) what changes in muscle structure occur, especially in relation to the capillaries, in chronic lung diseases? 5) is muscle blood flow and its distribution within muscle normal or not? and 6) how can these questions be best answered in intact humans with lung disease?

The physiological adaptations to exercise in chronic respiratory diseases were analysed by P. Palange (Rome, Italy). Exercise limitation in COPD is multifactorial in origin. The ability to increase ventilation during exercise is reduced; the more advanced the disease, the more impaired the exercise tolerance should be. However, factors other than ventilatory limitation play an important role in reducing exercise capacity in COPD [10]. In the advanced phase of the illness patients display variable degrees of peripheral muscle atrophy and muscle weakness has been reported to significantly contribute to exercise limitation [11]. At this stage patients reduce their daily activities to avoid exertional respiratory discomfort. Recent studies have demonstrated that the muscle aerobic capacity of stable hypoxaemic COPD patients is impaired. Slow $\mathrm{O}_{2}$ uptake kinetics at submaximal workloads have been reported [12]; ${ }^{31} \mathrm{P}-\mathrm{MRS}$ studies $[13,14]$ have shown that these patients rely heavily on nonaerobic sources to sustain moderate workloads; finally, early occurrence of metabolic acidosis has been demonstrated in patients with mild to severe COPD exercising at moderate intensity $[15,16]$. Oxygen supplementation may improve some of the derangements mentioned above $[5,17]$. The reduced ability to utilize the $\mathrm{O}_{2}$ transported by the circulation seems not to be related to reduction in bulk $\mathrm{O}_{2}$ flow [18] but to a defect in $\mathrm{O}_{2}$ transport and/or utilization at the tissue level. Several factors may contribute such as: 1) impaired diffusive conductance for $\mathrm{O}_{2}$ between RBCs and the mitochondria; 2) heterogeneous distribution of $\mathrm{O}_{2}$ bulk flow within the exercising muscle fibres; 3 ) inertia of the oxidative processes at the cellular level; 4) changes in distribution of muscle fibres and a reduction in muscle aerobic enzymes. P. Palange proposed to discuss the following arguments: influence of peripheral muscle dysfuntion on ventilatory demand during exercise; ventilatory response during different types of exercise; usefulness of "on-field" tests; oxygen consumption $\left(V^{\prime} \mathrm{O}_{2}\right)$ kinetics studies in the evaluation of peripheral muscle dysfunction.

The issue of muscle blood flow and $\mathrm{O}_{2}$ transport in chronic respiratory disorders was presented by J. Roca (Barcelona, Spain). Inability to increase ventilation and disturbances of pulmonary gas exchange have been historically invoked as principal determinants of exercise intolerance in COPD patients [19]. Different studies [9] indicate that these patients show a normal rise in cardiac output as $\mathrm{V}^{\prime} \mathrm{O}_{2}$ increases suggesting adequate ventricular function in uncomplicated disease. At peak exercise, cardiac output 
is low but it seems tightly coupled with ventilation and whole-body $V^{\prime} \mathrm{O}_{2}$. In athletes during extreme cycling exercise [20], regional blood flow distribution can be modulated by the perfusion requirements of the respiratory muscles such that blood flow to the exercising leg increases by alleviating the ventilatory load with noninvasive ventilation. It could be hypothesized that such a situation might also occur in severe COPD patients at a markedly lower maximal ventilation due to increased work of breathing. Moreover, low blood flow to the exercising legs can also be expected because of reduced muscle mass often seen in this disease [21]. Recent studies [18, $22]$, however, seem to confirm that muscle blood flow during exercise in COPD patients is preserved or even slightly increased. These patients show abnormally low mitochondrial oxidative capacity and low muscle bioenergetic status at a given submaximal exercise indicated by a higher inorganic phosphate concentration ([Pi])/phosphocreatine concentration $([\mathrm{PCr}])$ ratio and lower intracellular $\mathrm{pH}$ than healthy sedentary controls. Different lines of evidence suggest that intrinsic skeletal muscle dysfunction may be present in COPD patients and that it may play a role in the limitation of exercise tolerance. It is of note, however, that identification of skeletal muscle dysfunction in COPD can be obscured by several confounding factors, such as: 1) impaired muscle $\mathrm{O}_{2}$ transport; 2) steroid therapy; 3) detraining due to physical inactivity; or, 4) treatment with cyclosporine after lung transplantation.

In the discussion several issues were addressed, among them: $\mathrm{O}_{2}$ supply versus metabolic capacity as limits to exercise; relationship of symptoms to underlying physiolog$\mathrm{ical} /$ biochemical processes; and multiple factors in exercise limitation. Participants agreed on the need for future studies to rule out whether the skeletal muscle is not working properly because of structural and biochemical "intrinsic" defects or because the environment (e.g. hypoxia and microcirculation) in which the muscle works is altered. They also agreed on the need for studies in isolated small muscle groups to better evaluate the full oxidative capacity of the muscle; this is in fact difficult to ascertain with exercise protocols that involve large muscle groups (e.g. cycling) where exercise tolerance is limited by ventilatory and circulatory constraints.

\section{February 11, 1999. Section 2: structural and biochemical alterations of skeletal muscles in chronic respiratory diseases}

This section was opened by a short lecture by F. Maltais (Ste Foy, Canada) on "Skeletal muscle structure and function in chronic respiratory diseases". Peripheral muscle abnormalities described in patients with COPD include reduction in muscle mass and endurance, weakness, alteration in fibre type distribution, and decreased metabolic capacity $[16,23]$. Altered peripheral muscle function may also be present in other chronic respiratory disorders such as cystic fibrosis [24] and lung transplant recipients. The peripheral muscle changes in COPD are associated with an impaired muscle energy metabolism at rest and during exercise as indicated by nuclear MRS ${ }^{31} \mathrm{P}-\mathrm{NMR}$ ) studies [13]. An impaired oxidative phosphorylation and adenosine triphosphate (ATP) resynthesis with early activation of anaerobic glycolysis within the contracting muscles have been reported in these studies. These modifications in muscle energy metabolism do not appear to reflect a problem with peripheral perfusion since lower limb blood flow during exercise is similar between patients with COPD and normal subjects. Impaired muscle metabolism during exercise may affect exercise tolerance in chronic respiratory diseases by several mechanisms including enhanced lactic acidosis and ventilatory needs for a given exercise work rate [15] and premature muscle acidosis, a contributory factor to muscle fatigue and early exercise termination. Reduced peripheral muscle strength and endurance have also been associated with reduced exercise capacity in patients with COPD [25]. The nature of the skeletal muscle dysfunction in patients with COPD has not been fully elucidated. Several potential mechanisms may contribute to this problem and their relative importance is likely to vary from patient to patient. A unique mechanism explaining peripheral muscle dysfunction in all patients is unlikely. Chronic inactivity, nutritional imbalance, systemic steroids, hypoxaemia, a systemic inflammatory process with increased levels of blood cytokines, electrolyte disturbances, and low anabolic hormone levels have all been suggested as potential contributors to the development of peripheral muscle dysfunction in COPD.

A.M.W.J. Schols (Maastricht, The Netherlands) presented data on "Drug induced defects on skeletal muscles in chronic respiratory diseases". Investigation of possible drug induced defects in respiratory disease has been primarily focused on systemic glucocorticoid treatment. Indeed, evidence has been found [26] for an independent adverse effect of maintenance treatment with oral glucocorticoids on overall mortality in patients with COPD. This association is often automatically ascribed to steroid myopathy. This however has led to under exposure of the role of various other well documented side effects of sustained use of systemic corticosteroids including, in particular, suppression of the hypothalamic-adrenal axis, infectious complications and metabolic alterations. Functional and histological characteristics of peripheral muscle in COPD patients with clear steroid induced myopathy have been extensively described [26]. Patients suffering from peripheral muscle weakness and vastus lateralis biopsies were characterized by increased variation in diameter of fibres with several angular atrophic fibres and diffuse necrotic and basophilic fibres. On adenosine triphosphatase (ATPase) stain, a generalized fibre atrophy pattern was observed in contrast to experimental studies that have shown that steroids predominantly induce type IIb fibre atrophy. Nutritional depletion may also cause muscle wasting characterized by a more generalized fibre atrophy pattern. In a recent study (unpublished data) the skeletal muscle of patients with COPD, under long term low dose prednisolone treatment, were studied. Myopathic features in anterior tibialis muscle biopsies were mild and these morphological alterations were found both in COPD patients irrespective of steroid use as well as in healthy control subjects. Several other factors need to be considered for correct interpretation of steroid induced defects on skeletal muscle: dose and duration of steroid use, way of administration, steroid type, differences in contractile properties between nonfluorinated and fluorinated glucocorticoids at equipotent dose independent of muscle wasting per se, differences between in vitro contractile properties and in situ muscle function, differences between 
diaphragm and peripheral muscle. Limited clinical studies have investigated the effects of glucocorticoids on muscle metabolism. In a group of nondepleted patients with chronic bronchial asthma an increase in the synthesis and deltoid muscle cell deposition of glycogen and a decrease in the muscle glycogen degradation was found. Interestingly these findings were reported despite the absence of muscle weakness or morphological alterations [27]. Finally, it is still unclear how long steroid induced effects on muscle persist after systemic steroids are discontinued. This is clinically relevant since short courses of glucocorticoids are commonly prescribed during acute disease exacerbation.

P.D. Wagner introduced the issue of "Is there evidence for a specific myopathy in chronic respiratory diseases?" For many years, no attention was paid to the skeletal muscles as a possibly significant contributor to exercise limitation. This is no longer the case: more and more evidence is being collected that suggests myopathic involvement, especially in COPD. Most dramatic is the repeated finding that normalization of lung function by transplantation fails to restore maximal $V^{\prime} \mathrm{O}_{2}$ to more than $\sim 50 \%$ of normal, despite in some studies, inclusion of exercise rehabilitation programmes. This is clouded by the myopathic potential of immunosuppressives such as cyclosporin and steroids, but implies by exclusion that something may be intrinsically wrong with the muscles. In COPD oxidative enzyme levels appear reduced; blood lactate levels rise at lower power outputs than normal; there is greater intracellular acidosis and $\mathrm{PCr}$ depletion at a given power output than in healthy control subjects; $\mathrm{O}_{2}$ uptake and $\mathrm{PCr}$ recovery kinetics are slower in COPD as well. These all speak of metabolic insufficiency that limits exercise capacity. However, some of these factors are quite sensitive to $\mathrm{O}_{2}$ supply/muscle $\mathrm{Pa}, \mathrm{O}_{2}$ (which may themselves be abnormal in COPD), and also to fitness. Included are early lactate appearance, $\mathrm{PCr}$ recovery and depletion, and acidosis. Would these indices of apparent cellular pathology be normal when compared at similar intracellular $\mathrm{Pa}_{\mathrm{a}} \mathrm{O}_{2}$ and activity levels? Not all studies find reduced oxidative enzyme activity; in those that do, how much reduction does it take to interfere with maximal exercise capacity given that there probably is an excess of metabolic capacity in most normal people? Are such reductions pathological or just reflective of inactivity? Structural changes such as loss of type I muscle fibres, and reduction in the capillarity of muscles have been found. Similar questions apply here: what is the functional significance and pathological nature of the changes? Loss of muscle mass, difficulty in finding healthy control subjects with similar inactivity as patients, and variability in selection of patients for studies do not ease the interpretive problems. But perhaps most important is that most studies have asked COPD patients to engage in "whole body" (cycle) exercise that clearly pushes patients to ventilatory limits before their muscles have reached their maximal abilities: it is difficult to see how the capacity of the muscles can be assessed when they are shut down before reaching their potential. P.D. Wagner pointed out that cycle exercise may be very appropriate for many purposes, but probably not for study of the muscles themselves. Finally, the issue of regulation of exercise responses at the level of the gene is just beginning to be brought to bear on human disease. Studies at this level will be needed to understand mechanisms of muscle myopathy, if this syndrome indeed exists. P.D. Wagner then suggested the following focal points for discussion. What constitutes myopathy versus detraining versus $\mathrm{O}_{2}$ unavailability? How much change from normal must occur to have functional significance? Which metabolic parameters are $\mathrm{O}_{2}$-sensitive such that their interpretation requires comparison to healthy subjects at similar $\mathrm{O}_{2}$ levels? Choosing the proper control subjects. Can drugs used in lung disease account for the muscle structural and functional defects reported (immunosuppressives, steroids, bronchodilators)? Are the muscles in lung disease equally trainable as they are in health? What should be used to quantify effects of training?

At the end of the lectures the following issues were discussed: relationship of biochemical alterations to $\mathrm{O}_{2}$ availability; how much biochemical change from normal is of known significance; similarities and differences in skeletal muscles in other chronic diseases. Several questions were raised from the audience and some young researchers presented evidence on skeletal muscle dysfunction in chronic diseases other than COPD such as chronic heart failure and chronic renal failure. Participants agreed that comparisons with other diseases may help to answer the question, do COPD patients have a specific myopathy? They agreed on the need for future studies to rule out whether muscle dysfunction in COPD is simply the effect of ageing and reduced level of daily activity. Selection of appropriate control subjects appears to be crucial: body mass (and other anthropometric nutritional indices) and level of activity should be monitored in order to correctly answer this question.

\section{February 12, 1999. Section 3: effect of hypoxia and poor nutritional state on skeletal muscle function in chronic respiratory diseases}

This section was opened by a lecture on "The effect of hypoxia on muscle energetic" by X. Leverve (Grenoble, France). Muscle cells, like every other kind of cell, have to maintain energy homeostasis throughout many different problems, such as: 1) matching energy supply with energy demand; and 2) sharing ATP production among many pathways that compete for energy supply. But in these aspects, muscle cells appear to be very particular since they have to endure dramatic and major changes in ATP consumption rate according the state of contraction, as well as two further pathways: myofibrilar ATPases and endoplasmic-reticulum calcium uptake, both of which are very prominent in total cellular energy metabolism. Along with the different mechanisms of control, and regulation of the mitochondrial oxidative phosphorylation pathway, the role of the creatine kinase shuttle and the subsequent compartmentilization and channelling of energy transfer is probably of major importance in the determination of energy distribution and cellular priorities [28-31]. Therefore, by modulating the different isoforms of creatine kinase some adaptation to various energy status may occur.

Heart and skeletal muscles can also adapt to hypoxia, or to chronic energy limitation, by changing muscle contraction efficiency. As was reported in several models of ventricle hypertrophy, a change in the transcription of myosin isoforms can be linked to changes in contraction efficiency. Similar mechanisms are probably also involved in adaptation toward chronic hypoxia $[32,33]$. 
The concept of a "milieu interieur" as described by $\mathrm{C}$. Bernard, more than a century ago, or in modern words a "steady sate", is based on interorgan co-operation and competition. Hence, oxygen and nutrients or final waste products excepted, every metabolite can be a waste product for one cell and a substrate for an other one, either between different tissues or also between different cells in a single organ. Hence oxygen and glucose for instance must be shared between organs and cells. In this aspect the complete opposition between aerobic and anaerobic energy pathways is a very good example of alternative co-operation and competition: almost all organs compete for oxygen, most of them for glucose as an energetic substrate, insulin sensitivity being a major tool for tuning it, while other tissues or cells can oxidize lactate. Hence, lactate is either a final product or an energetic substrate. According to the fact that glucose or lactate in turn might be the actual substrate for the Kreb's cycle, there is a significant difference in the energy yield of oxygen reduction to water. Hence, in this manner some changes in lactate metabolism directly participates to the metabolic adaptation to hypoxia for all organs, including muscle and heart but probably in a different manner [34-36]. In this aspect, liver gluconeogenesis, which is affected by hypoxia, plays a role in peripheral tissues metabolism since the increased lactate concentration in plasma is probably responsible for an increased lactate use as oxidative energy substrate in some tissues (like peripheral muscle) rather than glucose [37, 38].

A.M.W.J. Schols introduced the issue of "Malnutrition in chronic respiratory diseases: the role of inflammation". There is increasing evidence for a role of inflammation in the pathogenesis of muscle wasting and cachexia in patients with chronic respiratory diseases. Experimental studies (unpublished data) have demonstrated an association between tumour necrosis factor (TNF)- $\alpha$ and weight loss in COPD [2]. Experimental animal and in vitro studies have shown that inflammation may affect both components of the energy balance, dietary intake on the one hand and energy and substrate metabolism on the other. Furthermore, direct effects of TNF- $\alpha$ on skeletal muscle have been implicated. Leptin, a protein synthesized by adipose tissue and encoded by the $o b$ gene, plays an important role in energy balance. Leptin is postulated to represent the afferent hormonal signal to the brain including the hypothalamus in a feedback mechanism regulating the fat mass. Three experimental studies have shown a link between inflammatory cytokines and leptin, leading to the hypothesis that adipose tissue gene expression is also influenced by inflammatory cytokines, which could induce anorexia in acute and chronic inflammation. Lep-tin levels are very low in emphysematous patients with low fat mass, in line with the reported feedback mech-anism in the regulation of fat mass. However in part of these patients elevated leptin levels adjusted for fat mass were found that were significantly associated with ele-vated plasma levels of soluble TNF-receptor 55 and a low dietary intake. Inflammation may also cause mobilization of skeletal muscle protein to meet the increased demand of amino acids for acute phase protein synthesis. Indeed COPD patients characterized by elevated levels of acute phase proteins were characterized by an elevated resting metabolic rate and depletion of fat-free mass, even out of proportion to the loss of body weight [39]. In line with the above proposed mechanisms, it is of interest to notice that normal weight COPD patients with depleted fat-free mass are characterized by a significantly lower peripheral muscle function and exercise performance than underweight COPD patients with preservation of fat-free mass. The latter finding also implies a direct effect of inflammation on skeletal muscle tissue. Studies in differentiated muscle cell lines indeed suggest that TNF- $\alpha$ directly induces skeletal muscle protein loss through reactive oxygen-mediated nuclear factor (NF)- $\kappa \mathrm{B}$ activation [40]. A.M.W.J. Schols concluded that further elucidation of the role of inflammation in pulmonary cachexia is essential from a therapeutic perspective since depleted patients that poorly respond to nutritional therapy have a worse prognosis than responders and are characterized by a systemic inflammatory response.

The effects of malnutrition on exercise capacity in chronic respiratory diseases were examined by P. Palange. Weight loss and poor nutritional status has been reported in $>25 \%$ of COPD patients [41, 42] with the largest incidence among patients with predominant emphysema. It is reasonable to assume that in malnourished individuals with COPD the energy-producing processes within the muscle may be less able to meet metabolic needs than in normally nourished, otherwise comparable patients. The possibility that malnutrition, leading to reduced intramuscular ATP turnover and a decrease in the concentration of phosphocreatine, may force the muscle to an early switch toward anaerobic glycolysis [43], has, however, been underestimated. In healthy humans, malnutrition reduces respiratory and peripheral muscle strength with limitation in exercise tolerance. Nutritional repletion improves strength and endurance of skeletal and respiratory muscles in underweight ambulatory COPD patients [44] and facilitates weaning from mechanical ventilation in COPD patients with respiratory failure [45]. Recent studies have demonstrated a positive correlation between nutritional state and maximal exercise tolerance as reflected by maximal $\mathrm{O}_{2}$ uptake $\left.[46,47]\right)$. In addition the findings of increased levels of wasted ventilation and high $\mathrm{O}_{2}$ cost of breathing in malnourished COPD [46] are compatible with the hypothesis that in these patients weight loss is caused by the caloric expenditures dictated by the high energy requirements of the respiratory muscles [48]. Recently a close correlation between nutritional state and the speed of $\mathrm{O}_{2}$ uptake kinetics during moderate exercise have been demonstrated in COPD patients, indicating that the decline in body mass parallels the impairment of muscle aerobic capacity [49]. The energy requirements of these patients must therefore be fulfilled via nonaerobic pathways, namely PCr breakdown and/or anaerobic glycolysis, even for light physical efforts. P. Palange pointed out that future studies are needed to validate the hypothesis that in malnourished patients with COPD a measurable enhancement of exercise tolerance can be achieved by rehabilitation programmes in conjunction with an adequate increase in caloric intake until body mass is recovered. P. Palange also proposed the following arguments to be discussed: mechanisms by which malnutrition leads to skeletal muscle dysfunction; can malnutrition be reversed and exercise capacity restored by nutritional and rehabilitation programmes?

R. Casaburi (Torrance, CA, USA) presented data on "Anabolic hormone deficiency and exercise intolerance in 
chronic respiratory diseases". There is a growing acceptance of the concept that dysfunction of the muscles of ambulation is responsible for a portion of the exercise intolerance seen in patients with chronic lung disease [1]. Muscles must have an adequate hormonal environment to grow and develop. Both the growth hormone and anabolic steroid systems act to improve muscle function. Recent studies have shown that plasma insulin-like growth factor (IGF)-1, (the principal mediator of growth hormone's action on muscle), and testosterone, (the predominant anabolic steroid in males), levels are low in COPD patients. Therefore, administering replacement doses of anabolic hormones would be rational therapy. Growth hormone supplementation has been shown to increase muscle mass, strength and endurance in growth hormone deficient adults. In studies of patients with chronic diseases, including COPD, growth hormone has yielded increases in muscle mass, though increases in functional exercise capacity have not generally been demonstrated. In hypogonadal males, replacement doses of testosterone substantially increase muscle mass and strength. Supraphysiological doses of testosterone have been shown to interact additively with strength training to yield impressive gains in muscle mass and strength in young healthy males [50]. Older males with mildly low testosterone levels have been shown to benefit as well [51]. Published studies indicate that COPD patients may benefit similarly [52]; additional studies are underway. Novel molecules targeted to yield specific anabolic effects in muscles are starting to be identified. R. Casaburi suggested that understanding of the cellular mechanisms of muscle growth must be improved so that interventions can be appropriately targeted. Also, researchers must learn to contrast the effects of anabolic interventions on strength versus endurance outcomes. This seems to be a fertile area for research.

During the discussion several topics were addressed, among them mechanisms of weight loss in COPD and the need to normalize exercise performance for muscle mass. The vast majority of the discussion, however was focused on the very interesting issue of metabolic abnormalities as the consequence of chronic hypoxaemia, particularly the effect of hypoxia on lactate turnover within the liver and other tissues. Participants were very attracted by the possibility suggested by the biochemist $X$. Leverve that muscle wasting could be the consequence of metabolic alteration and catabolic stimuli originating from tissues/organs different from the lung and the muscle itself (e.g. liver). The discussions were also kept alive by the very attractive issue that muscle damage in COPD may be secondary to a state of chronic inflammation; at this time, however, only few and incomplete data support this hypothesis.

February 12, 1999. Section 4: therapeutic interventions on skeletal muscle function in chronic respiratory disorders

This section was opened by J. Roca who introduced the issue of "The lessons learned from lung volume reduction". During the last few years, an increasing body of evidence indicate that lung volume reduction surgery (LVRS) can elicit significant short-term functional improvement in selected COPD patients. Different studies available provide valuable information on the underlying physiological mechanisms that explain the effects of this type of surgery $[53,54]$. Outcomes are defined in terms of: 1) changes in mortality; 2) improvement of health related quality of life; 3 ) exercise performance; and 4) physiological variables. Many questions, however, remain unresolved with respect to the long-term efficacy of the procedure. A critical problem is the lack of results of appropriate long-term randomized studies to assess outcome following LVRS. Data from the ongoing projects in this area may provide relevant information to evaluate the role of this surgical strategy. Descriptive data suggest that skeletal muscle impairment in COPD patients might play a role in the early and long-term outcome of this surgical procedure. On the other hand, LVRS provides a potentially unique situation to evaluate the role of peripheral muscle dysfunction in COPD patients since, in contrast to lung transplantation, potential confounding effects due to treatment with cyclosporine are not present.

A.G.N. Agusti presented data on "The lessons learned from lung transplantation". Traditionally, exercise limitation in patients with COPD has been explained on the basis of increased work of breathing and airflow limitation. According to this view, lung transplantation in COPD should normalize (or tend to normalize) both lung mechanics and exercise tolerance. However, this is not the case [55]. Despite marked improvement in functional outcome and quality of life after transplantation, available data show that the vast majority of patients with COPD still have persistent exercise limitation after surgery [56-59]. If it is considered that the central factors (lung mechanics, pulmonary gas exchange, pulmonary circulation) had been normalized (or tended towards normalization) after transplantation, this observation indicates that peripheral factors (oxygen transport and/or utilization by skeletal muscle) should be (remain?) abnormal in these patients. There is now evidence in the literature to support this contention. For instance, studies using NMR have shown abnormal skeletal muscle bioenergetics during exercise in these patients [59]. Potential mechanisms underlying this abnormality are varied and may include deconditioning and malnutrition, presence of a previous skeletal myopathy (systemic inflammation?) and/or use of drugs with the potential to interfere with mitochondrial metabolism, such as cyclosporin A [55]. A better understanding of these mechanisms might be relevant clinically because they constitute potential therapeutic targets [55].

R. Casaburi spoke on "The effects of training on skeletal muscle in chronic respiratory diseases". Exercise training is a key feature of rehabilitation programmes for patients with chronic respiratory disease. However, for many years it was unclear as to whether the benefits of training were primarily physiological (i.e., mediated by improvements in the function of the exercising muscles) or primarily psychological (e.g., a result of desensitization to dyspnoea).

It was previously believed that COPD patients could not achieve physiological training benefits because they were unable to exercise at work rates above their critical training intensity. However, it is now appreciated that these patients are able to exercise at a high fraction of their maximum power output. With this understanding, a series of investigations have provided unequivocal evidence that 
a rigorous exercise training regimen can induce beneficial changes in the muscles of ambulation [15, 22, 60, 61]. A lower level of blood lactate at a given level of exercise connotes better oxygen utilization by the muscles. More rapid oxygen uptake kinetics following exercise onset implies a smaller oxygen debt and better aerobic performance. Muscle biopsies have shown higher levels of aerobic enzymes and improved capillarity. Ongoing studies in several laboratories are investigating other manifestations of traininginduced improvements in muscle function.

The discussion was focused on possible therapeutic interventions aimed at ameliorating muscle performance and exercise tolerance in chronic respiratory disorders. Exercise training has been shown to reverse some of the metabolic abnormalities associated with deconditioning and ameliorate exercise tolerance. However, optimization of training programmes is needed. Participants agreed that in selected patients a promising approach is represented by the integration of surgical methods with muscle training.

\section{Conclusions}

The starting point for this workshop was the well known exercise intolerance in chronic lung diseases that for many years has been blamed on abnormal respiratory function, both lung mechanics and gas exchange. The principal overarching question posed was whether part of the blame lies with the locomotory muscles as well, and if so, how. Several lines of evidence do suggest that the muscles themselves may be structurally and functionally abnormal. However, such evidence, coming from morphological, biochemical and physiological measures, is itself somewhat difficult to interpret, so that while such abnormalities clearly exist, whether they reflect muscle abnormality per se or just the obligate muscle response to an abnormal physiological or biochemical environment (in terms of $\mathrm{O}_{2}$ transport, neural input, etc.) remains in contention. Part of the problem is what constitutes the most appropriate set of control subjects against which to compare exercise in patients with lung disease. Part of the problem resides in specific drug therapies (such as steroids and other immunosuppressive agents) that have myopathic effects themselves. Part of the problem results from difficulty separating effects of inactivity from those of disease per se since the two are usually closely linked. A specific additional layer of complexity comes from nutritional considerations and, in particular, loss of muscle mass which not infrequently accompanies severe disease and even greater exercise intolerance. Perhaps the essence of the overall problem can be seen from the generally poor posttransplantation exercise capacity of patients even many months post-surgery and after some degree of rehabilitation.

The consensus of the group is that the skeletal muscles are generally abnormal in chronic lung diseases. The group did know precisely in what functional respect, nor by what cellular and molecular mechanisms, nor how available therapies impinge on exercise capacity and muscle health.

These many uncertainties suggest that: 1) cellular and molecular level studies of muscle structure and function will be required to understand mechanisms; 2) study of small muscle groups that can exercise intensely (despite severe lung disease) is needed to identify the functional defects in muscle per se; 3 ) a similar comment applies to questions regarding trainability of muscle in these diseases; 4) functional studies of muscle contraction must consider the abnormal physiological environment in which the muscles exist, at the least, interpretation of function muscle allow for environmental abnormalities such as reduced blood flow or $\mathrm{O}_{2}$ transport; 5) the search for immunosuppressive molecules that do not cause myopathic changes should be a priority.

In conclusion, this workshop has laid out broad research directions to allow a better mechanistic understanding of muscle dysfunction in chronic lung diseases. Hopefully these will lead to therapeutic advances that will not only improve quality of life but also prolong it in the face of currently disappointing functional outcomes from the few available therapeutic modalities.

\section{References}

1. ATS/ERS Statement. Skeletal muscle dysfunction in obstructive pulmonary disease. Am J Respir Crit Care Med 1999; 159: S1-S40.

2. Koulouris NG, Dimopoulou I, Valta P, Finkelstein R, Cosio MG, Milic-Emili J. Detection of expiratory flow limitation during exercise in COPD patients. $J$ Appl Physiol 1997; 82: 723-731.

3. Wagner PD. Ventilation-perfusion inequality \& gas exchange during exercise in lung disease. In: Dempsey JA, Reed CE, eds. Muscular exercise and the lung. Madison, WI, USA, The University of Wisconsin Press, 1977; pp. 345-356.

4. Agusti AG, Roca J, Barbera JA, Casademont J, Rodriguez-Roisín R, Wagner $\mathrm{PD}$. Effect of sampling site on femoral venous blood gas values. J Appl Physiol 1994; 77: 2018-2022.

5. Palange P, Galassetti P, Mannix ET, et al. Oxygen effect on $\mathrm{O}_{2}$ deficit and $\mathrm{V}^{\prime} \mathrm{O}_{2}$ kinetics during exercise in obstructive pulmonary disease. $J$ Appl Physiol 1995; 78: 22282234.

6. Jakobsson P, Jorfeldt L. Nuclear magnetic resonance spectroscopy: a tool for skeletal muscle metabolic research. Eur Respir J 1992; 5: 151-154.

7. Punwani $\mathrm{S}$, Cooper CE, Clemence $\mathrm{M}$, et al. Correlation between absolute deoxyhaemoglobin $[\mathrm{dHb}]$ measured by near infrared spectroscopy (NIRS) and absolute R2' as determined by magnetic resonance imaging (MRI). $A d v$ Exp Med Biol 1997; 413: 129-137.

8. Sauleda J, Garcia-Palmer FJ, Wiesner R, et al. Cytochrome oxidase activity and mitochondrial gene expression in skeletal muscle of patients with chronic obstructive pulmonary disease. Am J Respir Crit Care Med 1998; 157: 1413-1417.

9. Agusti AGN, Cotes J, Wagner PD. Responses to exercise in lung diseases. In: Roca J, Whipp BJ, eds. Clinical exercise testing. Eur Respir Mon 1997; 6: 32-50.

10. Bauerle $\mathrm{O}$, Younes M. Role of ventilatory response to exercise in determining exercise capacity in COPD. $J$ Appl Physiol 1995; 79: 1870-1877.

11. Gosselink R, Troosters R, Decramer M. Peripheral muscle weakness contributes to exercise limitation in COPD. Am J Respir Crit Care Med 1996; 153: 976-980.

12. Nery LE, Wasserman K, Andrews JD, Huntsman DJ, Hansen JE, Whipp BJ. Ventilatory and gas exchange kinetics during exercise in chronic airways obstruction. $J$ Appl Physiol 1982; 53: 1594-1602. 
13. Kutsuzawa T, Shioya S, Kurita D, Haida M, Ohta Y, Yamabashi H. 31P-NMR study of skeletal muscle metabolism in patients with chronic respiratory impairment. Am Rev Respir Dis 1992; 146: 1019-1024.

14. Pajen JF, Wuyam B, Levy P, et al. Muscular metabolism during oxygen supplementation in patients with chronic hypoxemia. Am Rev Respir Dis 1993, 147: 592-598.

15. Casaburi R, Patessio A, Loli F, Zanaboni S, Donner CF, Wasserman K. Reductions in exercise lactic acidosis and ventilation as a result of exercise training in patients with obstructive lung disease. Am Rev Respir Dis 1991; 143: 9-18.

16. Maltais F, Simard AA, Simard C, Jobin J, Desgagnés P, Leblanc P. Oxidative capacity of the skeletal muscle and lactic acid kinetics during exercise in normal subjects and in patients with COPD. Am J Respir Crit Care Med 1996; 153: 288-293.

17. Mannix ET, Galassetti P, Boska MD, Burton G, Manfredi F, Farber M. $\mathrm{O}_{2}$ effect on ATP production in exercising muscle of COLD patients: a magnetic resonance spettroscopy study. J Appl Physiol 1995; 78: 2218-2227.

18. Maltais F, Jobin J, Sullivan MS, et al. Metabolic and hemodynamic response of lower limb during exercise in patients with COPD. J Appl Physiol 1998; 84: 1573-1580.

19. Gallagher CG. Exercise limitation and clinical exercise testing in chronic obstructive pulmonary disease. Clin Chest Med 1994; 15: 305-326.

20. Harms CA, Babcock MA, McClaran SR, et al. Respiratory muscle work compromises leg blood flow during maximal exercise. J Appl Physiol 1997; 82: 1573-1583.

21. Schols AMW, Slagen J, Volovics L, Wouters EFM. Weight loss is a reversible factor in the prognosis of chronic obstrcutive pulmonary disease. Am J Respir Crit Care Med 1998; 157: 1791-1797.

22. Sala E, Roca J, Marrades RM, et al. Effects of endurance training on skeletal muscle bioenergetics in chronic obstructive pulmonary disease. Am J Respir Crit Care Med 1999; 159: 1726-1734.

23. Schols AMWJ, Soeters PB, Dingemans MC, Mostert R, Frantzen PJ, Wouters EFM. Prevalence and characteristics of nutritional depletion in patients with stable COPD eligible for pulmonary rehabilitation. Am Rev Respir Dis 1993; 147: 1151-1156.

24. Lands LC, Heigenhauser GJF, Jones NL. Respiratory and peripheral muscle function in cystic fibrosis. $\mathrm{Am} R e v$ Respir Dis 1993; 147: 865-869.

25. Hamilton AL, Killian KJ, Summers E, Jones NL. Muscle strength, symptom intensity and exercise capacity in patients with cardiorespiratory disorders. Am J Respir Crit Care Med 1995; 152: 2021-2031.

26. Decramer M, de Bock V, Dom R. Functional and histologic picture of steroid-induced myopathy in chronic obstructive pulmonary disease. Am J Respir Crit Care Med 1996; 153: 1958-1964.

27. Fernandez-Sola J, Cusso R, Picado C, Vernet M, Grau JM, Urbano-Marquez A. Patients with chronic glucocorticoid treatment develop changes in muscle glycogen metabolism. J Neuro Sci 1993; 117: 103-106.

28. Saks VA, Khuchua ZA, Vasilyeva EV, Belikova O, Kuznetsov AV. Metabolic compartmentation and substrate channelling in muscle cells. Role of coupled creatine kinases in in vivo regulation of cellular respiration-a synthesis. Mol Cell Biochem 1994; 133: 155.

29. Saks VA, Tiivel T, Kay L, et al. On the regulation of cellular energetics in health and disease. Mol Cell Biochem 1996; 160: 195-208.

30. Brdiczka D, Wallimann T. The importance of the outer mitochondrial compartment in regulation of energy metabolism. Mol Cell Biochem 1994; 133: 69-83.

31. Kreher P, Keriel C, Leverve X. Action potentials in right and left ventricles from chronic hypoxic rats: effect of almitrine. Am J Physiol 1996; 271: H1856-H1863.

32. Pissaerek M, Bigard X, Mateo P, Guezennec CY, Hoerter JA. Adaptation of cardiac myosin and creatine kinase to chronic hypoxia. Am J Physiol 1997; 272: H1690-H1695.

33. Novel-Chaté V, Mateo P, Saks VA, Hoerter JA, Rossi A. Chronic exposure of rats to hypoxic environment alters the mechanism of energy transfer in myocardium. $J \mathrm{Mol}$ Cell Cardiol 1998; 30: 1295-1303.

34. McClelland GB, Hochachka PW, Weber JM. Carbohydrate utilization during exercise after high-altitude acclimation: a new perspective. Proc Natl Acad Sci USA 1998; 95: 10288-10293.

35. Hochachka PW, Clark CM, Holden JE, Stanley C, Ugurbil K, Menon RS. ${ }^{31} \mathrm{P}$ magnetic resonance spectroscopy of the Sherpa heart: a phosphocreatine/adenosine triphosphate signature of metabolic defense against hypobaric hypoxia. Proc Natl Acad Sci USA 1996; 93: 12151220.

36. Hochachka PW, Clark CM, Monge C, et al. Sherpa brain glucose metabolism and defense adaptations against chronic hypoxia. J Appl Physiol 1996; 81: 1355-1361.

37. Leverve X, Mustafa I, Péronnet F. Pivotal role of lactate in aerobic metabolism. In: Vincent $\mathrm{J}$, ed. Yearbook of intensive care and emergency medicine. Berlin, Germany, Springer Verlag, 1998: 588-596.

38. Pison CM, Chauvin C, Fontaine E, et al. Mechanism of gluconeogenesis inhibition in rat hepatocytes isolated af 268: E965-E973.

39. Schols AMWJ, Buurman WA, Staal-van den Brekel AJ, Dentener MA, Wouters EFM. Evidence for a relation between metabolic derangements and increased levels of inflammatory mediaters in a subgroup of patients with chronic obstructive pulmonary disease. Thorax 1996; 51 : 819-824.

40. Li Y, Schwartz RJ, Waddell IA, Holloway BR, Reid MB. Skeletal muscle myocytes undergo protein loss and reactive oxygen mediated NF- $\kappa \mathrm{B}$ activation in response to tumor necrosis factor $\alpha$. FASEB $J$ 1998; 12: 871-880.

41. Sahebjami H, Doers JT, Render ML, Bond TL. Anthropometric and pulmonary function test profiles of outpatients with stable chronic obstructive pulmonary disease. Am J Med 1993; 94: 469-474.

42. Wilson DO, Rogers RM, Hoffman RM. Nutrition and chronic lung disease. Am Rev Respir Dis 1985; 132: $1347-$ 1365.

43. Gertz I, Hedenstierna G, Hellers G, Wahren J. Muscle metabolism in patients with chronic obstructive lung disease and acute respiratory failure. Clin Sci Mol Med 1977; 52: 395-403.

44. Efthimiou J, Fleming J, Gomes C, Spiro GS. The effect of supplementary oral nutrition in poorly nourished patients with chronic obstructive pulmonary disease. Am Rev Respir Dis 1988; 137: 1075-1082.

45. Bassili HR, Deitel M. Effect of nutritional support on weaning patients off mechanical ventilators. JPEN 1981; 5: 161-163.

46. Palange P, Forte S, Felli A, Galassetti P, Serra P, Carlone $\mathrm{S}$. Nutritional state and exercise tolerance in patients with COPD. Chest 1995; 107: 1206-1212.

47. Baarends E, Schols AMWJ, Mostert R, Wouters EFM. Peak exercise response in relation to tissue depletion in patients with chronic obstructive pulmonary disease. Eur Respir J 1997; 10: 2807-2813. 
48. Mannix ET, Manfredi F, Palange P, Dowdeswell IRG, Farber M. Oxygen may lower $\mathrm{O}_{2}$ cost of ventilation in chronic obstructive lung diseases. Chest 1992; 101: 910915.

49. Palange $\mathrm{P}$, Forte $\mathrm{S}$, Onorati $\mathrm{P}$, et al. Effect of reduced body weight on muscle aerobic capacity in chronic obstructive pulmonary disease (COPD). Chest 1998; 114; 12-18.

50. Bhasin S, Storer TW, Berman N, et al. The effects of supraphysiologic doses of testosterone on muscle size and strength in normal men. $N$ Engl J Med 1996; 335: 1-7.

51. Tenover JS. Androgen administration to aging men. Endocrin Metab Clin N Am 1994; 23: 1092-1098.

52. Schols AMWJ, Soeters PB, Mostert R. Physiologic effects of nutritional support and anabolic steroids in patients with chronic obstructive pulmonary disease. $\mathrm{Am} \mathrm{J}$ Respir Crit Care Med 1995; 152: 1268-1274.

53. Benditt JOS, Lewis S, Wood DE, Klima L, Albert RK. Lung volume reduction surgery improves maximal $\mathrm{O}_{2}$ consumption, maximal minute ventilation $\mathrm{O}_{2}$ pulse, and dead space-to-tidal volume ratio during cycle ergometry. Am J Respir Crit Care Med 1997; 156: 561-566.

54. Sciurba FCR, Rodgers RM, Keenan RJ, et al. Improvement in pulmonary function and elastic recoil after lung-reduction surgery for diffuse emphysema. $N$ Engl $J$ Med 1996; 334: 1095-1099.

55. Estenne M. Higenbottam T. Exercise response in lung transplantation: assessment and rehabilitation programs.
In: Roca J, Whipp BJ, eds. Clinical exercise testing. Eur Respir Mon 1997; 6: 147-157.

56. Williams TJ, Patterson GA, McClean PA, Zamel N, Maurer JR. Maximal exercise testing in single and double lung transplant recipients. Am Rev Respir Dis 1992; 145: 101-105.

57. Ross DJ, Waters PF, Mohsenifar Z, Belman MJ, Kass RM, Koerner SK. Hemodynamic responses to exercise after lung transplantation. Chest 1993; 103: 46-53.

58. Ambrosino N, Bruschi C, Callegari G, et al. Time course of exercise capacity, skeletal and respiratory muscle perfomance after heart-lung transplantation. Eur Respir $J$ 1996; 9: 1508-1514.

59. Evans AB, Al-Himyary AJ, Hrovat MI, Papagianopoulos P, Wain JL, Ginns LC, Systrom DM. Abnormal skeletal muscle oxidative capacity after lung transplantation by 31P-MRS. Am J Respir Crit Care Med 1997; 155: 615621.

60. Casaburi R, Porszasz J, Burns MR, Chang RSY, Cooper CB. Physiologic benefits of exercise training in rehabilitation of patients with severe chronic obstructive pulmonary disease. Am J Respir Crit Care Med 1977; 155: 15411551.

61. Maltais F, Leblanc P, Simard C, et al. Skeletal muscle adaptation to endurance training in patients with chronic obstructive pulmonary disease. Am J Respir Crit Care Med 1996; 154: 442-447. 